\title{
ULTRA-SONOGRAFIA ENDOSCÓPICA (USE) DO ESÔFAGO, ESTÔMAGO, CÓLONS E RETO*
}

\author{
Márcio Martins Machado¹, Ana Cláudia Ferreira Rosa², Nestor de Barros³, Giovanni \\ Guido Cerri ${ }^{4}$
}

\begin{abstract}
Resumo Os autores relatam as principais utilizações da ultra-sonografia endoscópica nas afecções que envolvem o esôfago, estômago, cólons e reto. É relatada a metodologia para a realização do exame nesse setor do aparelho digestivo, indicando os principais achados. São descritos, ainda, alguns aspectos sonográficos à ultra-sonografia endoscópica de condições malignas e benignas do esôfago, estômago, cólons e reto. Unitermos:Ultra-sonografia. Ultra-sonografia endoscópica. Aparelho digestivo.
\end{abstract}

Abstract Endoscopic ultrasonography (EUS) of the esophagus, stomach, colon and rectum. The authors review the main applications of endoscopic ultrasonography in the management of diseases of the esophagus, stomach, colon and rectum. The methodology and the main findings at endoscopic ultrasonography are discussed as well as ultrasound findings of malignant and benign diseases of the esophagus, stomach, colon and rectum.

Key words: Ultrasonography. Endoscopic ultrasonography. Digestive tract

\section{INTRODUÇÃO}

A ultra-sonografia endoscópica (USE) é uma técnica que incorpora as imagens da ultra-sonografia convencional e os recursos visuais da endoscopia. Com esta combinação, auxiliada pelo uso de transdutores de alta frequiência, podem-se obter imagens de alta resolução. A USE permite que se estude a extensão das lesões nas paredes dos órgãos gastrointestinais, assim como auxilia na identificação de linfonodomegalias, tumores pancreáticos neuroendócrinos e cálculos biliares ${ }^{(1-4)}$.

* Trabalho realizado no Instituto de Radiologia (InRad) do Hospital das Clínicas da Faculdade de Medicina da Universidade de São Paulo (HC-FMUSP) e no Departamento de Radiologia do Hospital Sírio Libanês, São Paulo, SP.

1. Médico Radiologista do Hospital das Clínicas da Faculdade de Medicina da Universidade Federal de Goiás (UFG), Doutor em Radiologia pela FMUSP, Médico Radiologista Consultor do Departamento de Doenças do Aparelho Digestivo do Hospital Araújo Jorge (Hospital do Câncer) da Associação de Combate ao Câncer em Goiás, Membro Titular do Colégio Brasileiro de Radiologia (CBR).

2. Médica Radiologista do Hospital das Clínicas da Faculdade de Medicina da UFG, Pós-graduanda do Departamento de Radiologia daFMUSP, ex-Assistente do Departamento de Radiologia do Hospital Sírio Libanês,Membro Titular do CBR.

3. Professor Doutor do Departamento de Radiologia da FMUSP.

4. Professor Titular do Departamento de Radiologia da FMUSP, Chefe do InRad/HC-FMUSP, Diretor da Divisão de Diagnóstico por Imagem do Instituto do Coração (InCor) do HC-FMUSP, Diretor do Departamento de Radiologia do Hospital Sírio Libanês.

Endereço para correspondência: Dr. Márcio Martins Machado. Rua 1027, nำ230, Ed. Fabiana, apto. 304. Goiânia GO, 74823-120. E-mail: marciommachado@ibest.com.br

Recebido para publicação em 20/8/2001. Aceito, após revisão, em 19/11/2001
A USE também contribui no estudo das lesões de submucosa, dos tumores de esôfago e das neoplasias precoces de estômago. Ainda como possibilidades adicionais, temos o estudo das fístulas perianais e das lesões do aparelho esfincteriano anal.

Nesse contexto, é importante reconhecer os aspectos técnicos desse exame, assim como entender as características ultrasonográficas das principais doenças avaliadas, para que se implemente o diagnóstico multidisciplinar das doenças digestivas.

\section{INSTRUMENTAL E TÉCNICA}

Inicialmente, foram utilizados os transdutores lineares. Porém, foram os transdutores setoriais e radiais que adquiriram a preferência, por fornecerem um campo de imagem maior, o que facilita a orientação do examinador e torna os resultados mais reprodutíveis. Os transdutores radiais possibilitam a obtenção de imagens em 360 graus, em tempo real, o que facilita a avaliação das características anatômicas e patológicas das estruturas analisadas. Atualmente, os transdutores apresentam freqüências que variam de 7,5 a $12 \mathrm{MHz}$. Esta variação de freqüência permite que se controle a profundidade das imagens obtidas. Os transdutores lineares, por sua vez, apresentam importante utilidade na avaliação da extensão mural longitudinal dos tumores esofagianos ${ }^{(5,6)}$.
O estudo com ultra-sonografia depende da relação entre a penetração do feixe sonoro (que será maior, quanto menor for a frequiência do transdutor) e a capacidade de resolução da imagem (que será maior, quanto maior for a freqüência do transdutor). Com as frequiências maiores, perdese o poder de penetração do som, dificultando a avaliação de estruturas distantes, porém obtém-se maior resolução das imagens obtidas próximas do transdutor.

Dois tipos de instrumentos estão sendo empregados. Um deles utiliza o transdutor montado na extremidade do endoscópio $^{(\mathbf{7 , 8})}$. O outro transdutor endoscópico utilizado, o "mini-probe", apresenta o formato dos cateteres que habitualmente são usados nos procedimentos endoscópicos. Este transdutor é passado pelo canal do endoscópio de uso rotineiro, realizando-se, então, o estudo ecográfico ${ }^{(\mathbf{9 , 1 0})}$.

Os transdutores convencionais comercialmente disponíveis habitualmente apresentam freqüências variando de 7,5 a 12 MHz. Já os transdutores ("mini-probes") que são passados pelos canais de biópsias dos endoscópios apresentam frequiências variando de 12 a $20 \mathrm{MHz}^{(5)}$. O recurso do Doppler também pode ser encontrado em aparelhos mais modernos, maximizando a dinâmica do estudo ultra-sonográfico ${ }^{(6)}$.

Para se obter interface adequada para a passagem do som, pode-se realizar a infusão de líquido, ficando o ecoendoscópio 
imerso nesse meio líquido. No estudo do estômago, duodeno e reto pode ser empregada esta técnica. Em outras situações os ecoendoscópios apresentam, em suas extremidades, balões que podem ser insuflados com líquido (água), fornecendo a interface adequada. Dentre as estruturas avaliadas por este método temos o esôfago, o estômago, o duodeno, os cólons e o reto, assim como as estruturas que se situam próximas a eles ${ }^{(4)}$.

A USE também pode ser usada pela via endorretal e colonoscópica, favorecendo o estudo do canal anal, reto, dos cólons e de estruturas adjacentes, como os linfonodos. Como em outras partes do aparelho digestivo, o reto foi inicialmente avaliado com transdutores lineares, sendo necessária a rotação manual do transdutor em 360 graus para a análise de toda a circunferência. Posteriormente, foram utilizados os transdutores com maior campo de imagem, como os radiais, que avaliam uma maior área, facilitando o estudo. Recentemente, foram desenvolvidos ecoendoscópios para serem usados na avaliação de todo o cólon. Um dos aparelhos disponíveis tem transdutor de 7,5 MHz, com um campo de imagem de 320 graus. Para a obtenção da interface adequada pode ser usado também o método com balão ou a técnica de imersão do transdutor em meio líquido ${ }^{(4,11)}$.

Com instrumentos mais recentes poderse-ia ainda implementar o estudo das lesões parietais e extraluminais, incluindo os linfonodos, por meio da realização de biópsias aspirativas com agulha fina ${ }^{(6)}$. Estainovação vem sendo adicionada à USE.

\section{CAMADAS DO APARELHO DIGESTIVO AVALIADAS PELA ECOENDOSCOPIA}

As paredes do trato gastrointestinal, do ponto de vista da ecoendoscopia, são divididas em cinco camadas (Figura 1). A primeira camada, hiperecogênica, corresponde à borda ecogênica entre o transdutor e a mucosa superficial. A segunda camada, hipoecogênica, corresponde à mucosa e à muscular da mucosa. A terceira camada, hiperecogênica, corresponde à submucosa. A quarta camada, hipoecogênica, corresponde à muscular própria. A quinta camada, hiperecogênica, corresponde à se-

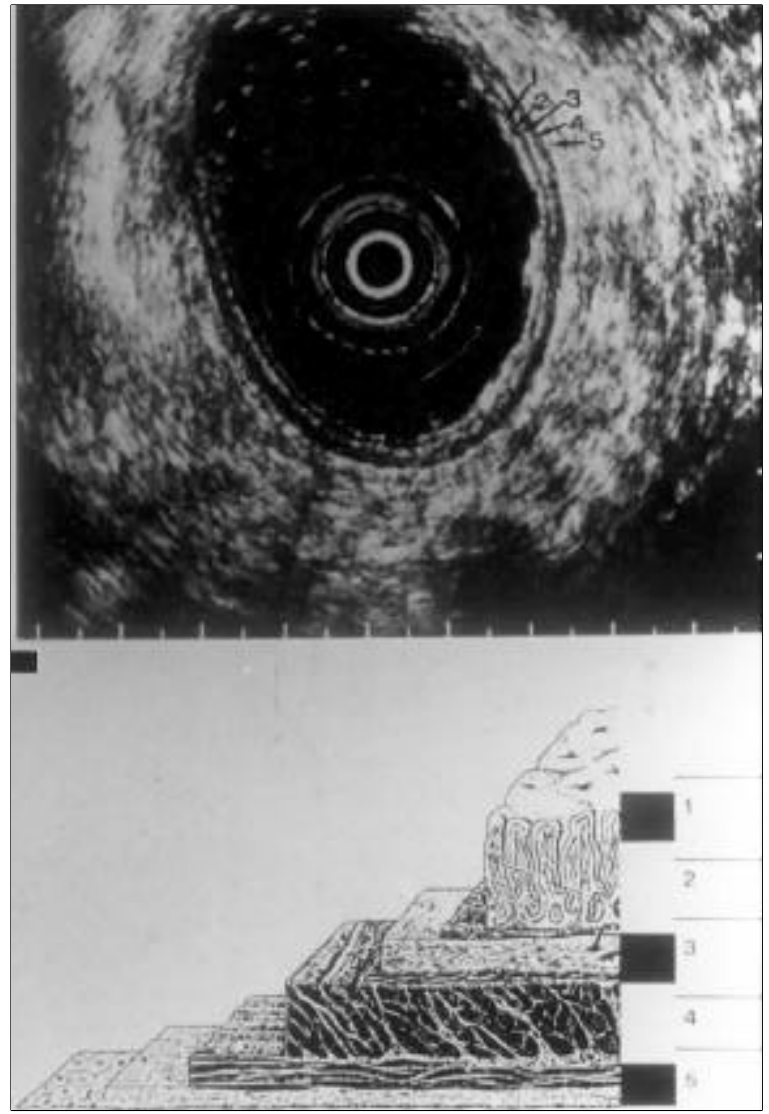

Figura 1. Anatomianormal-camadas das paredes do trato gastrintestinal. 1 - Primeira camada: hiperecogênica, corresponde à borda ecogênica entre o transdutor e a mucosa superficial; 2 - segunda camada: hipoecogênica, corresponde à mucosa e muscular da mucosa; 3 - terceira camada: hiperecogênica, corresponde à submucosa; 4 - quarta camada: hipoecogênica, corresponde à muscular própria; 5 - quinta camada: hiperecogênica, corresponde à serosa ou à subserosa, ou nos casos dos órgãos extraperitoneais, à adventícia.

rosa, ou à subserosa, ou nos casos dos órgãos extraperitoneais, à adventícia ${ }^{(3,5)}$.

\section{ESÔFAGO}

Embora no aparelho digestivo, como referido anteriormente, tenhamos usualmente cinco camadas à ecoendoscopia, o esôfago pode apresentar-se, muitas vezes, com três camadas. O esôfago apresenta-se dessa maneira pelo fato da sua parede não estar na zona de foco do aparelho, ou pelo fato do balão deslocar a parede do esôfago axialmente, afastando-a do transdutor. Seu estudoé melhor realizado pela técnica que utiliza o balão insuflado com água. No caso de serem identificadas apenas três camadas em sua parede, elas seriam: a primeira camada, hiperecogênica, corresponde à mucosa-submucosa, sendo que a segunda camada, hipoecogênica, corresponde à muscular própria, e a terceira camada se apresenta hiperecogênica e corresponde à adventícia (a interface entre a muscular própria e os tecidos periesofagianos) $)^{(\mathbf{4})}$. Evidentemente, com ajustes adequados, podemos demonstrar aquelas cinco camadas descritas anteriormente.

No esôfago, podemos avaliar o carcinoma esofagiano. $\mathrm{O}$ exame permite que seja identificada a posição da lesão, tomando como referência a distância entre o tumor e a arcada dentária superior, da mesma forma que é realizado habitualmente pela endoscopia. Pode ser também estudada a profundidade da invasão tumoral na parede do esôfago e sua extensão longitudinal. Entretanto, esses dados devem ser avaliados com cautela, na medida em que a acurácia da USE, para esses dois últimos fatores, pode variar de $72 \%$ a $92 \%{ }^{(6,12)}$.

Sonograficamente, o carcinoma esofagiano apresenta-se como imagem hipoecogênica (Figura 2) que altera a anatomia sonográfica habitual da parede do esôfago. Um ponto de importância no planejamento do tratamento clínico-cirúrgico é estabelecer as relações da lesão com a aorta e a traquéia. A identificação de invasão destas estruturas denota doença avançada e sem possibilidade curativa, embora procedimentos paliativos possam ser usados ${ }^{(4)}$. 
Outra característica avaliada pela USE é a identificação de linfonodos metástaticos. Neste sentido, a USE apresenta acurácia variando de $71 \%$ a $88 \%$. O próprio tamanho dos linfonodos deve ser analisado com cuidado, uma vez que linfonodos com $3 \mathrm{~mm}$ a $5 \mathrm{~mm}$ de tamanho já podem estar envolvidos por metástases. Ainda com relação ao estudo dos linfonodos, a acurácia da USE em identificar linfonodos não-metastáticos é baixa, menor que $56 \%$, devido principalmente à dificuldade de se diferenciar linfonodos com micrometástases (menores que $5 \mathrm{~mm}$ ) daqueles normais ou inflamatórios ${ }^{(4,6,11)}$. Não obstante, a USE apresenta maior precisão do que outros exames pré-operatórios naidentificação da infiltração parietal e na identificação de linfonodos metastáticos nos casos de carcinomas do esôfago ${ }^{(4,6,11)}$.

As lesões submucosas também podem ser avaliadas pela USE. A USE pode identificar o local de origem dessas lesões, como de submucosa (no caso dos lipomas) e de muscular própria (no caso de leiomiomas e leiomiossarcomas). Permite, ainda, que compressões extrínsecas (como no caso de linfonodos mediastinais) sejam prontamente identificadas, afastando-se a possibilidade de causas intramurais ${ }^{(\boldsymbol{6})}$.

\section{ESTÔMAGO E DUODENO}

O estômago normalmente é avaliado pela técnica de "imersão", podendo asso- ciar-se também a insuflação do balão. $\mathrm{O}$ duodeno pode ser avaliado tanto pela técnica da "imersão", quanto pela técnica do balão. Com esse estudo, podem seridentificadas facilmente as cinco camadas. No estômago, merece destaque o fato de que a quarta camada apresenta-se especialmente exuberante no antro e piloro. A espessura habitual da parede gástrica, embora sujeita a variação, pode ser considerada como sendo de até $3 \mathrm{~mm}$ no estômago bemdistendido $^{(\mathbf{1 1})}$.

O estômago pode ser utilizado como janela para a avaliação das estruturas retroperitoneais e intra-abdominais. Neste sentido, o fígado pode ser avaliado pela pequena curvatura gástrica e pelo duodeno. O baço é avaliado através da porção alta da pequena curvatura. $O$ pâncreas pode ser visualizado através da parede posterior do corpo gástrico, observando-se sua relação com a veia esplênica. Em algumas situações, a cauda e o corpo pancreáticos são melhor avaliados pelo fundo/corpo gástrico. A análise cuidadosa da cabeça pancreática pode ser estabelecida ao nível do duodeno, observando-se, como ponto de reparo, a confluência entre as veias porta e esplênica $^{(4)}$.

O estudo dos carcinomas gástricos é extremamente importante, pois somente o diagnóstico precoce permite expectativa de cura maior. Sobrevidas de $90 \%$ têm sido referidas para casos precoces submetidos a gastrectomias curativas ${ }^{(\mathbf{1 3})}$. O carcinoma gástrico precoce é aquele que acomete apenas a mucosa ou a submucosa, independentemente do comprometimento linfonodal metastático regional ${ }^{(14)}$. A USE auxilia na identificação dessas lesões precoces, contribuindo no planejamento cirúrgico.

Na USE, o carcinoma gástrico se apresenta como lesão hipoecogênica, que modifica o padrão habitual de cinco camadas das paredes gástricas (Figura 3). A acurácia da USE em precisar a invasão parietal varia de $73 \%$ a $84 \%$. Apresenta especial dificuldade a diferenciação dos tumores que invadem a muscular própria e a subserosa, daqueles que penetram a serosa mas não invadem estruturas adjacentes. O envolvimento metastático dos linfonodos regionais pode ser identificado com precisão em $68 \%$ a $80 \%$ dos casos. Já a identificação de linfonodos não-metastáticos é menos precisa, ocorrendo em menos de $56 \%$ dos $\operatorname{casos}^{(\mathbf{4 , 1 5}, 16)}$.

A USE não permite diferenciar com precisão úlceras benignas de malignas, mas auxilia no estudo de lesões diagnosticadas (por biópsias) como malignas, avaliando a extensão locorregional dos tumores ${ }^{(16)}$.

Os tumores gástricos recidivados apresentam dificuldade em serem diagnosticados pelos exames pré-operatórios, como a tomografia computadorizada e a ultra-sonografia. Essa dificuldade é compartilhada também pela USE, uma vez que é difícil diferenciar as alterações inflamatórias das recidivas tumorais ${ }^{(\mathbf{3}, \mathbf{4}, \mathbf{1 7})}$.

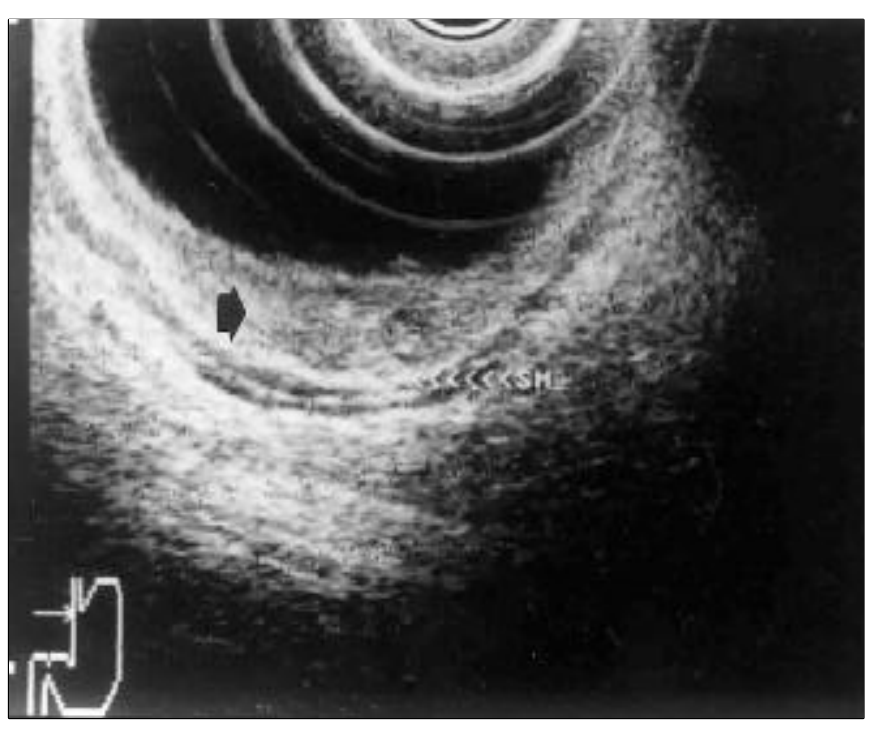

Figura 2. Câncer de esôfago: lesão superficial (seta) com invasão até a submucosa. Observar o aspecto hipoecogênico e heterogêneo da lesão.

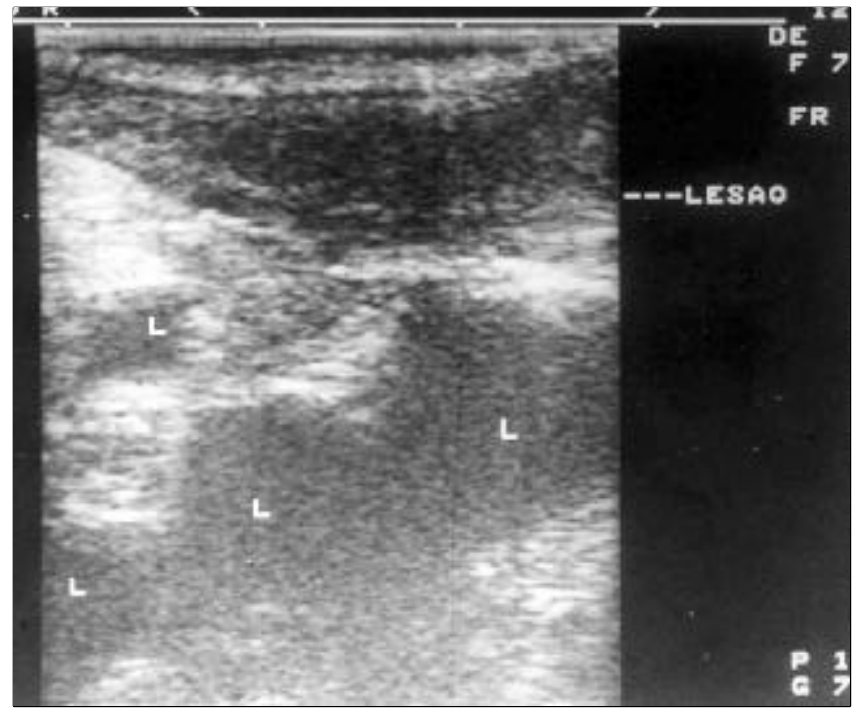

Figura 3. Adenocarcinoma gástrico envolvendo toda a espessura da parede (lesão), com linfonodos adjacentes (L). 
Os linfomas gástricos devem ser estudados com cautela. É sabido que, especialmente nas fases iniciais, esses tumores envolvem as paredes gástricas (especialmente a lâmina própria e a submucosa), mas preservam a superfície epitelial da mucosa. Por isso, muitas vezes eles não são diagnosticados nem mesmo pela endoscopia digestiva alta com a realização de biópsias. A análise das paredes gástricas nos linfomas mostra que o envolvimento ocorre principalmente pela disseminação longitudinal, diferentemente do carcinoma gástrico, no qual ela é, principalmente, em profundidade. Na USE observa-se espessamento da segunda e terceira camadas. $\mathrm{Na}$ eventualidade de se encontrar esse espessamento de maneira importante, associado com ulceração da mucosa, a possibilidade de ser linfoma é bastante provável. Novamente, deve ser notado que nas fases iniciais podemos encontrar apenas espessamento da segunda e terceira camadas, preservando a estratificação habitual da parede gástrica em cinco camadas, e sem a presença de ulceração na mucosa. Em casos mais avançados encontramos lesão de aspecto difuso e hipoecogênico, transmural, perdendo-se a estratificação em camadas, refletindo o envolvimento de todos os níveis da parede gástrica ${ }^{(\mathbf{4 , 1 8})}$. Em outros casos, os linfomas apresentam-se como agregados de múltiplos nódulos hipoecogênicos, murais ${ }^{(5)}$.

Pregas gástricas exuberantes também podem ser estudadas pela USE, e algumas generalizações podem auxiliar no diagnóstico. Pregas gástricas exuberantes, associadas com espessamento local das camadas da parede gástrica, usualmente ocorrem em doenças benignas, como na gastrite, úlcera péptica e síndrome de Zollinger-Ellison. Já o espessamento difuso das pregas gástricas com perda da estratificação em camadas sugere linfoma ou linite plástica (carcinoma gástrico infiltrativo). Pregas proeminentes, associadas com imagens hipoecogênicas/anecóicas na parede gástrica, sugerem varizes submucosas ${ }^{(\mathbf{1 9})}$.

Podem ser referidos, ainda, o estudo de lesões parietais e a diferenciação entre elas e as compressões extrínsecas. Os tumores musculares (leiomiomas e leiomiossarcomas) podem ocorrer em outras partes do aparelho digestivo, mas serão discutidos nessa sessão de estômago, onde são encontrados com maior frequiência. Podem ser identificados na quarta camada (originados da muscular própria) e também na segunda camada (originados na muscular da mucosa). Os leiomiomas tendem a se apresentar hipoecogênicos, menores que $4 \mathrm{~cm}$, com margem bem delimitada. Já os leiomiossarcomas, embora se apresentem predominantemente hipoecogênicos, apresentam aspecto um pouco mais heterogêneo, identificando-se áreas ora mais, ora menos ecogênicas, podendo ser observadas áreas anecóicas de permeio. Com relação às dimensões, usualmente apresentam mais de $4 \mathrm{~cm}$ de diâmetro. Tanto os leiomiomas quanto os leiomiossarcomas podem apresentar ulceração no topo da lesão ${ }^{(4)}$.

Já os lipomas são identificados habitualmente como lesões hiperecogênicas, com margens bem delimitadas, originando-se da submucosa, podendo ocorrer também em outras localidades do aparelho digestivo. O pâncreas ectópico, usualmente identificado no antro, apresenta-se como lesão hipoecogênica, podendo apresentar imagem ductular anecóica no seu interior. Habitualmente, são identificados na terceira camada (submucosa). As varizes gástricas apresentam-se como imagens serpiginosas, anecóicas, usualmente submucosas. Entretanto, elas podem ser extramurais, perfurantes e intramurais ${ }^{(\mathbf{4 , 5})}$.

As compressões extrínsecas podem ser reconhecidas pela USE, na medida em que as camadas parietais apresentam-se preservadas, observando-se a massa compressiva extragástrica ${ }^{(5)}$.

Os carcinóides, nos estágios iniciais, comumente se apresentam cobertos por mucosa íntegra. À ecoendoscopia, esses tumores têm aspecto homogêneo, hipoecogênico, identificados com maior frequiência na terceira camada. Ocasionalmente, esses tumores apresentam aspecto ecográfico mais variado, com áreas mais ecogênicas, dependendo das características histológicas da lesão ${ }^{(20)}$.

Os cistos são identificados como imagens anecóides, localizadas comumente na segunda e terceira camadas, apresentando margens bem definidas. Os linfangiomas apresentam-se também como lesões anecóicas, estendendo-se pela mucosa e sub$\operatorname{mucosa}^{(5)}$.

\section{CÓLON E RETO}

Como nas outras partes do aparelho digestivo, encontramos cinco camadas ecogenicamente distintas. No reto, a quarta camada ecográfica apresenta-se especialmente evidente, representando a camada muscular, que anatomicamente se divide em camadas circular interna e longitudinal externa.

Os tumores colorretais representam importante causa de morbidade e mortalidade dentre as neoplasias do aparelho digestivo. Devido a este fato, os métodos diagnósticos devem contribuir para aprimorar seu estadiamento. As neoplasias colorretais apresentam-se, à USE, como lesões hipoecogênicas. Podemos encontrar áreas mais ecogênicas, representando calcificações ou fibrose $e^{(3,4)}$.

As técnicas cirúrgicas de ressecção de tumores retais, com preservação do aparelho esfincteriano, necessitam de uma acurada avaliação pré-operatória com relação à invasão parietal pelo tumor e do comprometimento dos músculos esfincterianos. A USE endorretal e endoanal contribui nesse sentido, auxiliando no planejamento cirúrgico. Os casos com doença mais localizada, com margem de ressecção livre de tumor e sem envolvimento do aparelho esfincteriano, poderiam ser operados de maneira radical, mas preservando-se o aparelho esfincteriano (assoalho pélvico e canal anal) ${ }^{(3)}$. Deste modo, os pacientes continuariam evacuando pelo ânus, sem a necessidade de colostomia permanente.

Outro aspecto a ser considerado é a identificação de linfonodos metastáticos regionais. No câncer do reto a USE pode orientar a realização de procedimentos mais conservadores, como a ressecção local (no caso de lesões localizadas, pequenas e sem linfonodos comprometidos). Entretanto, deve ser lembrado, enfaticamente, que os fatores que orientam a realização das ressecções locais dos tumores retais são variados (tamanho e diferenciação do tumor, mobilidade da lesão em relação à parede do reto ao toque retal) e não apenas relacionados ao envolvimento metastático dos linfonodos do mesorreto ${ }^{(3)}$.

Muitos estudos têm demonstrado sensibilidade e especificidade de aproximadamente $90 \%$ na avaliação da extensão do 
envolvimento da parede retal pelo tumor ${ }^{(3)}$ A identificação de linfonodos metastáticos apresenta precisão variável, comíndices de $74 \%$, segundo alguns autores ${ }^{(3)}$. Deve ser notado que existem algumas dificuldades em se afirmar o comprometimento metastático de linfonodos, especialmente aqueles pequenos (menores que $1,0 \mathrm{~cm}$ ).

Mesmo tumores dos cólons têm sido avaliados pela USE, corroborando para o diagnóstico e estudando a presença de linfonodomegalia locorregional $^{(\mathbf{4})}$. Deve-se ressaltar que muitos autores ponderam sobre a dificuldade de se diferenciar linfonodos metastáticos dos inflamatórios ${ }^{(\mathbf{1 , 2})}$.

Outras alterações podem ser avaliadas pela USE colorretal. Recidivas locais dos tumores retais podem ser estudadas, embora possa ser difícil a diferenciação com alterações fibróticas pós-operatórias. O estudo das paredes do cólon também pode ser realizado nas doenças inflamatórias (retocolite ulcerativa idiopática, Crohn), identificando as alterações parietais decorrentes do processo inflamatório, embora os achados não sejam específicos ${ }^{(4)}$.

As alterações do aparelho esfincteriano decorrentes de lesões traumáticas, os abscessos e as fístulas também podem ser estudados pela USE. Este método diagnóstico auxiliaria na identificação dos traje- tos fistulosos, das cavidades abscedidas e dos pontos de lesão esfincteriana, orientando a terapêutica ${ }^{(17)}$.

\section{REFERÊNCIAS}

1. Jochem RJ, Reading CC, Dozois RR, Carpenter HA, Wolff BG, Charboneau JW. Endorectal ultrasonographic staging of rectal carcinoma. Mayo Clin Proc 1990;65:1571-7.

2. Roubein LD, David C, DuBrow R, et al. Endoscopic ultrasonography in staging rectal cancer. Am J Gastroenterol 1990;85:1391-4.

3. Corman ML. Carcinoma of the rectum. In: Corman ML, ed. Colon and rectal surgery. 3rd ed. Philadelphia, PA: JB Lippincott, 1993:596-720.

4. Torres GM. Endoscopic ultrasonography. In: Gore RM, Levine MS, Laufer I, eds. Textbook of gastrointestinal radiology. 1st ed. Philadelphia, PA. WB Saunders, 1994:127-39.

5. Chonan A, Mochizuki F, Ando M. Endosonography. In: Margulis AR, Baert AL, Brandy WL, eds. Modern imaging of the alimentary tube. 1st ed. New York, NY: Springer-Verlag, 1998:62-70.

6. Botet JF, Gerdes H. Endoscopic ultrasound of the gastrointestinal tract. In: Kane RA, Donley S, eds. Intraoperative, laparoscopic and endoluminal ultrasound. 1st ed. Philadelphia, PA: Churchill Livingstone, 1999:169-83.

7. Strohm WD, Phillip J, Hagenmüller F, Classen M. Ultrasonic tomography by means of an ultrasonic fiberendoscope. Endoscopy 1980;12:2414.

8. DiMagno EP, Buxton JL, Regan PT, et al. Ultrasonic endoscope. Lancet 1980;1:629-31.

9. Silverstein FE, Martin RW, Kimmey MB, Jiranek GC, Franklin DW, Proctor A. Experimental evaluation of an endoscopic ultrasound probe: in vitro and in vivo canine studies. Gastroenterology 1989; 96:1058-62.
10. Kimmey MB, Martin RW, Silverstein FE. Endoscopic ultrasound probes. Gastrointest Endosc 1990;36(2 Suppl):S40-6.

11. Botet JF, Lightdale CJ. Endoscopic sonography of the upper gastrointestinal tract. AJR 1991;156: 63-8.

12. Rosch T. Experience with staging of esophageal cancer. In: Endoscopic ultrasonography: a tutorial. Cleveland, $\mathrm{OH}$ : Cleveland Clinic Foundation, 1991:113-24.

13. Abe $\mathrm{S}$, Ogawa $\mathrm{Y}$, Nagasue $\mathrm{N}$, et al. Early gastric cancer: results in a general hospital in Japan. World J Surg 1984;8:308-14.

14. Japanese classification of gastric cancer. Japanese Research Society for Gastric Cancer. Tokyo, Japan: Kanehara \& Co., 1995.

15. Lightdale CJ. EUS staging of gastric cancer. In Endoscopic ultrasonography: a tutorial. Cleveland, OH: Cleveland Clinic Foundation, 1991: 219-31.

16. Rosch T. Staging stomach cancer by EUS: clinical usefulness. In: Endoscopic ultrasonography: a tutorial. Cleveland, $\mathrm{OH}$ : Cleveland Clinic Foundation, 1991:205-14.

17. Corman ML. Anorectal abscess and anal fistula. In: Corman ML, ed. Colon and rectal surgery. 3rd ed. Philadelphia, PA: JB Lippincott, 1993:13387.

18. Bolondi L, Casanova P, Caletti GC, Grigioni W, Zani L, Barbara L. Primary gastric lymphoma versus gastric carcinoma: endoscopic US evaluation. Radiology 1987;165:821-6.

19. Botet JF. Endoscopic US in the evaluation of patients with large gastric folds. Radiology 1990; 177(P): 115

20. Yoshikane H, Tsukamoto Y, Niwa Y, et al. Carcinoid tumors of the gastrointestinal tract: evaluation with endoscopic ultrasonography. Gastrointest Endosc 1993;39:375-83. 